\title{
Surface acoustic wave mediated exciton dissociation in a $\mathrm{ZnCdSe} / \mathrm{LiNbO}_{3}$ hybrid
}

Cite as: Appl. Phys. Lett. 94, 193505 (2009); https://doi.org/10.1063/1.3136862

Submitted: 26 February 2009 • Accepted: 26 April 2009 • Published Online: 14 May 2009

D. A. Fuhrmann, A. Wixforth, A. Curran, et al.

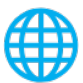

\section{ARTICLES YOU MAY BE INTERESTED IN}

Surface acoustic wave regulated single photon emission from a coupled quantum dotnanocavity system

Applied Physics Letters 109, 033105 (2016); https://doi.org/10.1063/1.4959079

Independent dynamic acousto-mechanical and electrostatic control of individual quantum dots in a $\mathrm{LiNbO}_{3}-\mathrm{GaAs}$ hybrid

Applied Physics Letters 106, 013107 (2015); https://doi.org/10.1063/1.4905477

Exciton transport in strained monolayer $\mathrm{WSe}_{2}$

Applied Physics Letters 113, 252101 (2018); https://doi.org/10.1063/1.5063263

四 QBLOX

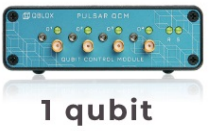

Shorten Setup Time Auto-Calibration More Qubits

Fully-integrated Quantum Control Stacks Ultrastable DC to $18.5 \mathrm{GHz}$ Synchronized $<<1 \mathrm{~ns}$ Ultralow noise

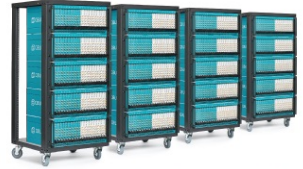

100s qubits

visit our website > 


\title{
Surface acoustic wave mediated exciton dissociation in a $\mathrm{ZnCdSe} / \mathrm{LiNbO}_{3}$ hybrid
}

\author{
D. A. Fuhrmann ${ }^{1,2}$ A. Wixforth, ${ }^{1,2}$ A. Curran, ${ }^{3}$ J. K. Morrod, ${ }^{3}$ K. A. Prior, ${ }^{3}$ \\ R. J. Warburton, ${ }^{2,3}$ and J. Ebbecke ${ }^{2,4, a)}$ \\ ${ }_{1}^{1}$ Institut für Physik der Universität Augsburg, Experimentalphysik I, Universitätsstr. 1, \\ 86159 Augsburg, Germany \\ ${ }^{2}$ Center for NanoScience (CeNS), Geschwister-Scholl-Platz 1, 80539 Munich, Germany \\ ${ }^{3}$ School of Engineering and Physical Sciences, Heriot-Watt University, Edinburgh EH14 4AS, \\ United Kingdom \\ ${ }^{4}$ University of Southern Denmark, Mads Clausen Institute, NanoSYD, Alsion 2, \\ DK-6400 Sonderborg, Denmark
}

(Received 26 February 2009; accepted 26 April 2009; published online 14 May 2009)

\begin{abstract}
By making use of epitaxial lift-off, $\mathrm{ZnCdSe}$ quantum wells are transferred onto a $\mathrm{LiNbO}_{3}$ substrate in order to employ its enhanced piezoelectric properties. The photoluminescence emission of this hybrid structure is characterized and the influence of a surface acoustic wave on the free exciton and bound exciton emission is investigated. Finally, two counterpropagating surface acoustic waves are launched leading to a decrease in the acoustic wave mediated exciton dissociation. (C) 2009 American Institute of Physics. [DOI: 10.1063/1.3136862]
\end{abstract}

In GaAs/AlGaAs quantum wells (QWs), excitons have a binding energy of approximately $5 \mathrm{meV}$ causing the photoluminescence $(\mathrm{PL})$ spectrum to be dominated by excitonic recombinations at low temperatures. The binding energy of an exciton is related to the oscillator strength and depends on how well the electron and hole wave functions are matched. The exciton binding energy in II/VI-semiconductors is, for example, in $\mathrm{ZnCdSe} \mathrm{QWs}$, up to $35 \mathrm{meV}$ due to an increased oscillator strength and excitonic recombinations can be detected almost up to room temperature. This increased exciton binding energy is one main difference and also one advantage in comparison to the GaAs/AlGaAs-heterostructures and has opened up single quantum dot emission at elevated temperatures. ${ }^{1}$ Additionally, $\mathrm{ZnSe}$ is piezoelectric for certain crystal directions but as for GaAs the coupling coefficients are small compared to materials such as $\mathrm{LiNbO}_{3}$. A decade ago, it was discovered that the piezoelectric field accompanying a propagating surface acoustic wave (SAW) can exceed the exciton binding strength in GaAs/AlGaAs QWs and can lead to a SAW mediated exciton dissociation. ${ }^{2-4}$ These electrons and holes can be transported by the SAW and by incorporating single quantum emitters first experimental results toward a SAW mediated single photon source were reported. ${ }^{5}$

In this paper, acousto-optic effects in $\mathrm{ZnCdSe}$ QWs will be presented and due to the increased exciton binding energy an improved sample layout was necessary in order to increase the piezoelectric fields for the SAW mediated exciton dissociation. Therefore, $\mathrm{ZnCdSe}$ QWs were removed from the host substrate and transferred to a $\mathrm{LiNbO}_{3}$ substrate using an epitaxial lift-off technique which was originally developed for $\mathrm{GaAs} / \mathrm{AlGaAs}$-heterostructures. ${ }^{6}$ A schematic sketch of the sample layout and liftoff technique is presented in Fig. 1.

Initially, a $73 \mathrm{~nm}$ buffer layer of ZnSe:Mn was deposited on a $n^{+}$-doped GaAs substrate followed by a sacrificial layer

${ }^{a)}$ Electronic mail: ebbecke@mci.sdu.dk.
(60 nm) of MgS. ${ }^{7}$ This was followed by $63 \mathrm{~nm}$ of $\mathrm{ZnSe}$ and five QWs $\left(\mathrm{Zn}_{0.9} \mathrm{Cd}_{0.1} \operatorname{Se} 4 \mathrm{~nm} / \mathrm{ZnSe} 8 \mathrm{~nm}\right)$ which were capped with a $53 \mathrm{~nm}$ thick $\mathrm{ZnSe}$ layer. For the epitaxial liftoff, small pieces of this heterostructure were covered with apiezon wax. Dilute $\mathrm{HCl}$ was used to selectively remove the sacrificial $\mathrm{MgS}$ layer ${ }^{8}$ and the QWs were transferred to the $\mathrm{LiNbO}_{3}$ substrate where interdigital transducers (IDTs) had been processed (see Fig. 1). Finally, the wax was removed in a solvent and the samples were cleaned in de-ionized water. The IDTs on the $\mathrm{LiNbO}_{3}$ substrate were used to launch SAWs with large amplitude. In order to investigate acoustooptic effects in this hybrid $\mathrm{ZnCdSe} / \mathrm{LiNbO}_{3}$ system a circular spot of laser excitation is focused by a lens to approximately $5 \mu \mathrm{m}$ in diameter. The same lens was used to collect the radiation of recombination. A schematic sketch of the detection setup is shown in Fig. 1 (not to scale).

Due to the increased exciton binding energy in this II/VI material the excitonic emission can be detected at higher temperatures than in GaAs. Examples of these spectra are presented in Fig. 2(a). The main peak is the free exciton (FX) recombination and by temperature and laser power dependence studies the low energy shoulder was identified to be the bound exciton (BX) emission. From the distance between the main peak and the BX peaks a binding energy to the impurity of $\approx 7 \mathrm{meV}$ can be estimated but at present it is not possible to determine if the excitons are bound to donors or

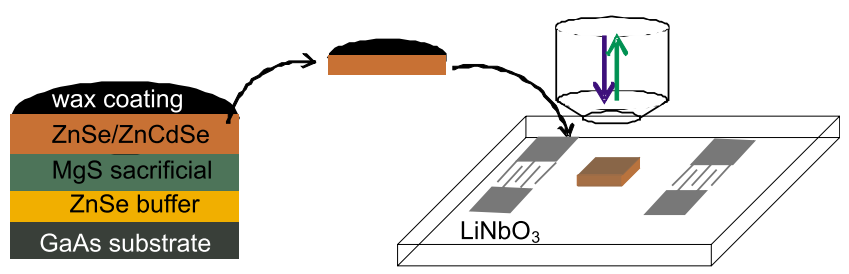

FIG. 1. (Color online) Schematic sketch of the epitaxial liftoff technique and the measurement setup: $\mathrm{ZnCdSe} \mathrm{QWs}$ were grown on a GaAs substrate on top of a sacrificial $\mathrm{MgS}$ layer. Using a selective etchant solution the $\mathrm{MgS}$ layer can be removed and the top layers were transferred onto a $\mathrm{LiNbO}_{3}$ substrate with prestructured IDTs. 


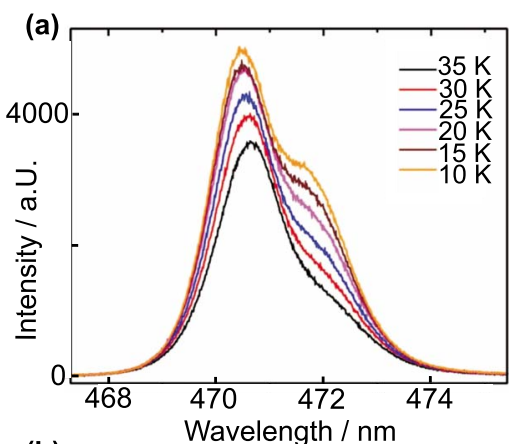

(b)

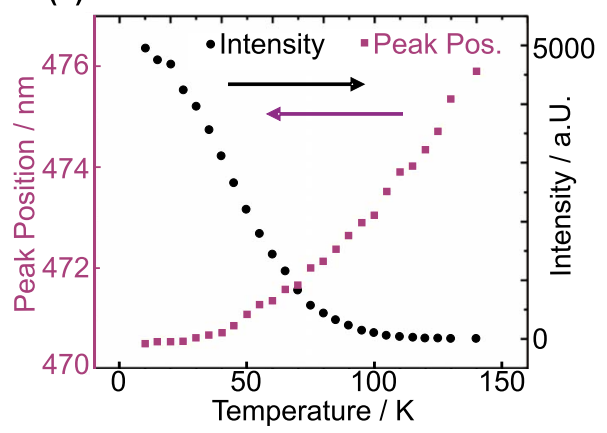

FIG. 2. (Color online) (a) Temperature dependence of the PL intensity: In (b) the peak position of the FX emission and the associated PL intensity are presented.

acceptors. Besides the decrease in the PL intensity of the BXs also a shift in the emission wavelength is visible. This shift is analyzed in Fig. 2(b) at temperatures between 5 and $140 \mathrm{~K}$. The emission wavelength is altered by the temperature change from approximately $470 \mathrm{~nm}$ at $5 \mathrm{~K}$ to $476 \mathrm{~nm}$ at $130 \mathrm{~K}$ and is caused by the energetic bandgap decrease due to the thermal expansion of the material. Additionally, the thermal energy of the excited electron-hole-pairs is increased leading to a decrease in the detected PL intensity by increased thermal dissociation. Also the interaction with optical phonons decreases the PL intensity at elevated temperatures causing the disappearance of the PL signal at temperatures above $T \approx 145 \mathrm{~K}$.

In Fig. 3 the SAW mediated PL quenching in the $\mathrm{ZnCdSe} \mathrm{QWs}$ at a temperatures of $6.5 \mathrm{~K}$ is presented. If the piezoelectric SAW field becomes larger than the exciton oscillator field strength then electron-hole-pairs are dissociated. Additionally, the SAW spatially modulates the conduction and valence bands of the semiconducting material which leads to a spatial separation of electrons and holes, as sketched in Fig. 3(a). The wave function overlap is strongly reduced resulting in a decrease in the PL signal; this is the SAW mediated PL quenching. The electric field necessary for the dissociation depends on the exciton oscillator strength and therefore this hybrid system $\mathrm{ZnCdSe} / \mathrm{LiNbO}_{3}$ was developed. On $\mathrm{LiNbO}_{3}, \mathrm{SAWs}$ with much larger amplitude can be launched due to the increased electromechanical coupling factor $K^{2}$. In Fig. 3(b) measurements at $T=6.5 \mathrm{~K}$ and a SAW wavelength of $\lambda=v / f=3940 \mathrm{~m} / \mathrm{s} / 112.5 \mathrm{MHz}=35 \mu \mathrm{m}$ are presented and show that the SAW can quench the PL signal for large rf power levels $P \geq 10 \mathrm{dBm}$. Due to the large exciton binding energy such large rf power levels are necessary even on the piezoelectric material $\mathrm{LiNbO}_{3}$. In order to exclude thermal effects a pulsed measurement setup is used. Figure 3(c) presents the integrated PL intensity of the FX main peak and the $\mathrm{BX}$ shoulder as a function of rf power

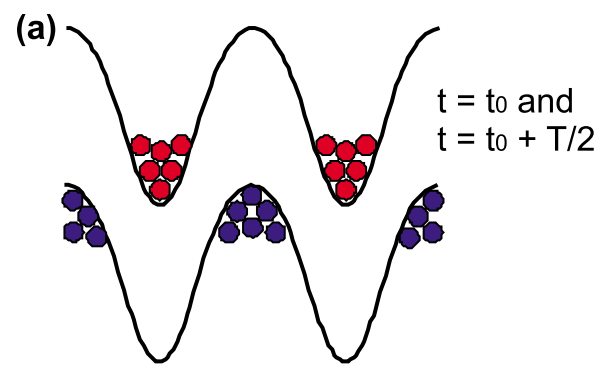

(b)
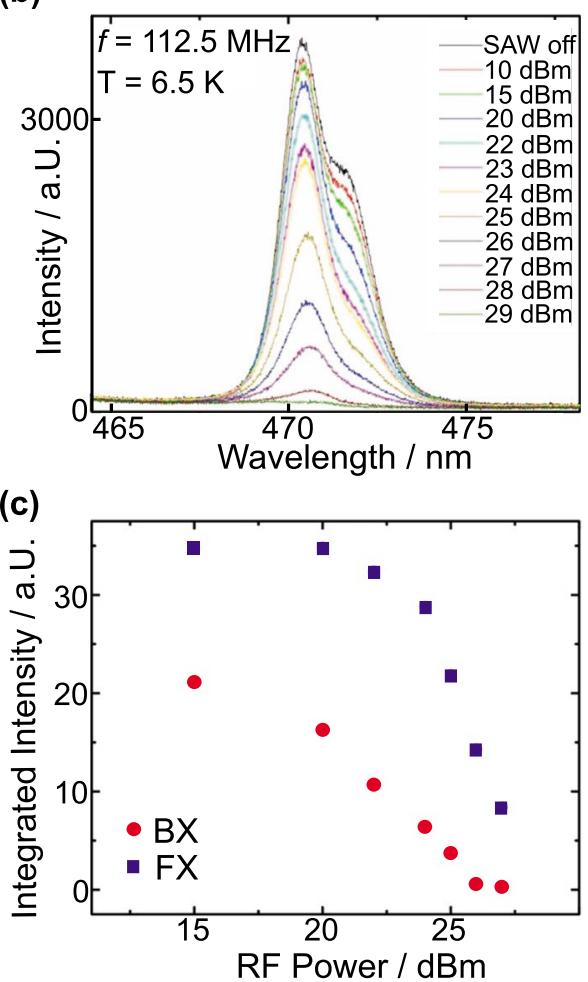

FIG. 3. (Color online) (a) Schematic sketch of the QW band edges modulated by a propagating SAW. Laser excited electrons and holes relax to the band extrema and are therefore spatially separated. (b) Decrease in the excitonic emission at $T=6.5 \mathrm{~K}$. In (c) the integrated PL intensity of the FXs and bound excitonic contributions are shown as a function of RF power employing the data of (b).

using the data of Fig. 3(b) and for a dissociation of the BX a lower SAW power is required than for the FXs in accordance with the estimated binding energies.

In Fig. 3 it was shown that a SAW can dissociate excitons in the $\mathrm{ZnCdSe}$ QWs. Furthermore, the SAW propagates out of the focus of the lens and drags the charge carriers away in its potential minima. ${ }^{2-4}$ This bipolar charge transport was first published in Ref. 2. There, an additional metal stripe at the sample surface was used to screen the piezoelectric fields of the SAW. The band edges became flat and a PL signal at the metal stripe was detected caused by the resulting wave function overlap of the transported electrons and holes. Effectively, light was transported by the SAW and the point of release was determined by the position of the metal stripe.

An alternative method of delocalized recombination can be employed which is based on the additional launching of a second SAW. Two counterpropagating SAWs with identical frequency and amplitude create a standing wave with nodes and antinodes formed with a spacing of $\lambda / 2$ caused by the 

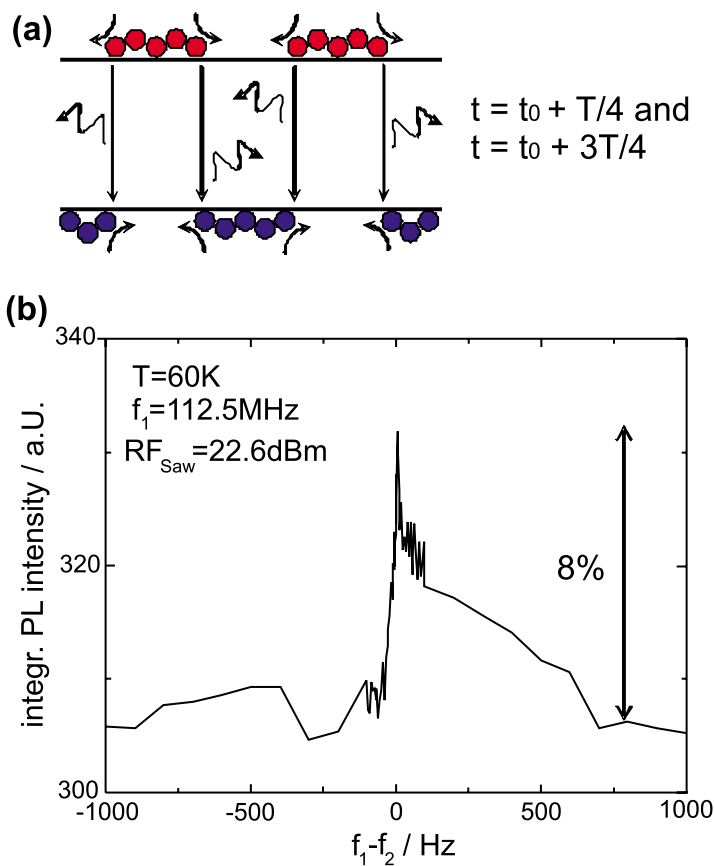

FIG. 4. (Color online) Schematic sketch of the QW band edges for a standing SAW in the case of destructive surface wave interference (adapted from Ref: 9). For a short time period the band edges are flat and the charge carriers form excitons and recombined. (b) Experimental verification that in the case of a standing SAW the exciton dissociation is decreased and therefore the integrated PL intensity increases. Note the narrow frequency interval of standing wave condition.

fact that the two SAWs interfere constructively and destructively in a temporally alternating way. For the short time period of destructive interference the energy bands are completely flat [see sketch in Fig. 4(a) in comparison to Fig. $3(\mathrm{a})$ ] and there is a finite probability of electron and hole recombination if the exciton lifetime is in the order of this time interval. This situation is sketched in Fig. 4(a) and was adapted from Refs. 2 and 9 who were able to show a decreased PL quenching by the use of two counterpropagating SAWs in GaAs QWs.

In Fig. 4(b) the integrated PL intensity evolution is presented for such a standing wave measurement in $\mathrm{ZnCdSe}$ QWs. The intensity was measured as a function of frequency difference between counterpropagating SAWs of frequency $f_{1}$ and $f_{2}$. There was an increase in the PL intensity detected for a frequency interval of only a few tenths of Hertz, more than six orders of magnitude smaller than the SAW frequency. In this narrow frequency region a standing wave is formed. Therefore, a decrease in PL intensity quenching was detected mediated by a standing SAW.
The measurement of the decreased SAW mediated exciton dissociation of Fig. 4(b) can be employed to estimate the FX lifetime. The integrated PL intensity without applying a standing SAW was 650 a.u. in comparison to approximately 310 a.u. with applying a SAW [see Fig. 4(b)] which means that the signal increases by $8 \%$ to its original value when applying a standing SAW. This increase can be used to calculate the exciton lifetime: $0.92=\exp (-\Delta t / \tau)$ with $\tau$ as the FX lifetime and $\Delta t$ as the time interval where the flatband condition is fulfilled. By using the approximation that a SAW can be described by a trigonometric function the flatband condition is fulfilled twice for $2 \%$ of each wave cycle. The period of a SAW with frequency of $112.5 \mathrm{MHz}$ is $8.9 \mathrm{~ns}$ which leads to a time period of $\Delta t=50 \mathrm{ps}$. This results in an estimation of the FX lifetime of $\tau=600$ ps which is roughly in the same order of magnitude as previously measured lifetimes of $100 \mathrm{ps}$ in ZdCdSe QWs. ${ }^{10,11}$

In summary, we have investigated the influence of a SAW on the characteristic PL of a ZnCdSe QW. For these experiments, the QWs were transferred onto a $\mathrm{LiNbO}_{3}$ substrate by epitaxial lift-off in order to make use of the enhanced piezoelectric properties of this hybrid structure. The experiments presented with the $\mathrm{ZnCdSe} / \mathrm{LiNbO}_{3}$ hybrid system are encouraging and further detailed investigations of acoustodynamic effects have been initiated.

This work was supported in part by the German government through the Cluster of Excellence "NIM." Two of the authors (K.A.P. and R.J.W.) like to thank the EPSRC for financial support.

${ }^{1}$ R. Arians, A. Gust, T. Kümmell, C. Kruse, S. Zaitsev, G. Bacher, and D. Hommel, Appl. Phys. Lett. 93, 173506 (2008).

${ }^{2}$ C. Rocke, S. Zimmermann, A. Wixforth, G. Böhm, and G. Weimann, Phys. Rev. Lett. 78, 4099 (1997)

${ }^{3}$ C. Rocke, A. O. Govorov, A. Wixforth, G. Böhm, and G. Weimann, Phys. Rev. B 57, R6850 (1998).

${ }^{4}$ P. V. Santos, M. Ramsteiner, and F. Jungnickel, Appl. Phys. Lett. 72, 2099 (1998).

${ }^{5}$ C. Bödefeld, J. Ebbecke, J. Toivonen, M. Sopanen, H. Lipsanen, and A. Wixforth, Phys. Rev. B 74, 035407 (2006).

${ }^{6}$ E. Yablonovitch, Phys. Rev. Lett. 58, 2059 (1987).

${ }^{7}$ C. Bradford, C. B. O'Donnell, B. Urbaszek, A. Balocchi, C. Morhain, K. A. Prior, and B. C. Cavenett, J. Cryst. Growth 227, 634 (2001).

${ }^{8}$ A. Balocchi, A. Curran, T. C. M. Graham, C. Bradford, K. A. Prior, and R. J. Warburton, Appl. Phys. Lett. 86, 011915 (2005).

${ }^{9}$ H.-J. R. Kutschera, dissertation, LMU Munich (2003).

${ }^{10}$ E. Runge, A. Schülzgen, F. Henneberger, and R. Zimmermann, Phys. Status Solidi B 188, 547 (1995) (b).

${ }^{11}$ W. Heiss, G. Prechtl, D. Stifter, H. Sitter, G. Springholz, T. Riemann, F. Bertram, D. Rudloff, J. Christen, G. Bley, U. Neukirch, J. Gutowski, and J. Liu, Appl. Phys. Lett. 75, 974 (1999). 